\title{
GENERALIZED SHEAR DEFORMATIONS FOR ISOTROPIC INCOMPRESSIBLE HYPERELASTIC MATERIALS
}

\author{
JAMES M. HILL
}

(Received 27 January 1977)

(Revised 18 March 1977)

\begin{abstract}
For isotropic incompressible hyperelastic materials the single function characterizing generalized shear deformations or as they are sometimes called anti-plane strain deformations must satisfy two distinct partial differential equations. Knowles [5] has recently given a necessary and sufficient condition for the strain-energy function of the material which if satisfied ensures that the two equations have consistent solutions. It is shown here for the general material not satisfying Knowles' criterion that the only possible consistent solutions of the two partial differential equations are those which are already known to exist for all strain-energy functions. More general types of generalized shear deformations for such materials are shown to exist only for special or restricted forms of the strain-energy function. In deriving these results we also obtain an alternative derivation of Knowles' criterion.
\end{abstract}

\section{Introduction}

Generalized shear deformations which are sometimes referred to as anti-plane strain deformations are characterized by a single function. For isotropic incompressible hyperelastic materials this function must satisfy two generally distinct partial differential equations. Knowles [5] has recently obtained a necessary and sufficient condition which ensures that every solution of one of these partial differential equations is automatically a solution of the second equation. There are, however, many standard strain-energy functions proposed for rubber-like materials (see, for example, [1], [2] and [3]) which do not satisfy Knowles' criterion.

(C) Copyright Australian Mathematical Society 1978

Copyright. Apart from any fair dealing for scholarly purposes as permitted under the Copyright Act, no part of this JOURNAL may be reproduced by any process without written permission from the Treasurer of the Australian Mathematical Society. 
The purpose of this paper is to investigate possible consistent solutions of these equations for materials of the latter type. We show for the general strain-energy function not satisfying Knowles' criterion that the only possible solutions are those which are known to exist for all strain-energy functions (namely those given by (2.7)). More general types of deformations are shown to exist only for special or restricted forms of the strain-energy function. In establishing these results we also obtain an alternative derivation of the necessary and sufficient condition given in [5].

In the following section we give the basic equations for generalized shear deformations of isotropic incompressible hyperelastic materials. In Section 3 we give an alternative derivation of the sufficiency of the criterion (2.8) due to Knowles [5] which follows immediately from (3.9). For those materials not satisfying Knowles' criterion we use (3.9) in Section 4 to show not only the necessity of (2.8) but also that the only generalized shear deformations which apply to all strain-energy functions are those given by (2.7). In Section 5 we discuss briefly the possibility of obtaining more general types of deformations for particular forms of the strain-energy function. The first case considered is interesting since the restriction obtained on the strain-energy function is a generalization of that given in [5]. Moreover, for this case the mathematical details can be effected in simple terms so as to obtain an explicit form for the deformation.

\section{Terminology and basic equations}

For material and spatial rectangular cartesian coordinates $(X, Y, Z)$ and $(x, y, z)$ respectively, static generalized shear deformations are given by

$$
x=\lambda^{-t} X, \quad y=\lambda^{-t} Y, \quad z=\lambda Z+u(X, Y),
$$

where $\lambda$ is a positive constant and $u(X, Y)$ is a function of $X$ and $Y$ only. If for isotropic incompressible hyperelastic materials we use the general equations given in Hill [4] for the stress and strain tensors and the equilibrium equations, then for (2.1) we can show in a straightforward manner that the basic equations for $u(X, Y)$ are given by

$$
\begin{gathered}
{\left[\phi_{1}(\omega) u_{X}\right]_{X}+\left[\phi_{1}(\omega) u_{Y}\right]_{Y}=0,} \\
q_{X}=\left[\phi_{2}(\omega) u_{X}^{2}\right]_{X}+\left[\phi_{2}(\omega) u_{X} u_{Y}\right]_{Y}, \\
q_{Y}=\left[\phi_{2}(\omega) u_{Y}^{2}\right]_{Y}+\left[\phi_{2}(\omega) u_{X} u_{Y}\right]_{X},
\end{gathered}
$$

where subscripts denote partial differentiation, $q(X, Y)$ is related to the usual arbitrary pressure function for incompressible materials and the function $\omega(X, Y)$ is defined by

$$
\omega^{2}=u_{X}^{2}+u_{Y}^{2}
$$


The response coefficients $\phi_{1}(\omega)$ and $\phi_{2}(\omega)$ are defined in terms of the strain-energy function of the material $\Sigma\left(I_{1}, I_{2}\right)$ by

$$
\phi_{1}(\omega)=\frac{\partial \Sigma}{\partial I_{1}}+\frac{1}{\lambda} \frac{\partial \Sigma}{\partial I_{2}}, \quad \phi_{2}(\omega)=\frac{\partial \Sigma}{\partial I_{2}},
$$

where $I_{1}$ and $I_{2}$ are the invariants of the strain tensor of (2.1) which can be shown to be given by

$$
I_{1}=\lambda^{2}+\frac{2}{\lambda}+\omega^{2}, \quad I_{2}=\frac{1}{\lambda^{2}}+2 \lambda+\frac{\omega^{2}}{\lambda},
$$

and we remind the reader that the dependence of $\phi_{1}$ and $\phi_{2}$ on the constant $\lambda$ will not be noted explicitly.

If we eliminate $q(X, Y)$ from (2.2.2) and (2.2.3) by equating expressions for $q_{X Y}$ then we obtain

$$
\frac{\partial^{2}}{\partial X \partial Y}\left[\phi_{2}(\omega)\left(u_{X}^{2}-u_{Y}^{2}\right)\right]=\left(\frac{\partial^{2}}{\partial X^{2}}-\frac{\partial^{2}}{\partial Y^{2}}\right)\left[\phi_{2}(\omega) u_{X} u_{Y}\right]
$$

and this equation and (2.2.1) are the two partial differential equations which the function $u(X, Y)$ must satisfy. We remark that (2.6) is satisfied identically for all strain-energy functions if $u(X, Y)$ takes on any of the following forms:

(i) $u(X, Y)=A \tan ^{-1}(Y / X)+B$,

(ii) $u(X, Y)=A X+B Y+C$,

(iii) $u(X, Y)=\phi\left(X^{2}+Y^{2}\right)$,

(iv) $u(X, Y)=\psi(A X+B Y)$,

where $A, B$ and $C$ denote arbitrary real constants and $\phi$ and $\psi$ are functions of the arguments indicated. We note that the deformations (i) and (ii) are controllable while for the deformations (iii) and (iv) the functions $\phi$ and $\psi$ depend upon the strain-energy function of the material. For all of the above deformations we can always find a function $q(X, Y)$ such that (2.2.2) and (2.2.3) are satisfied. Knowles [5] has shown that a necessary and sufficient condition for every solution of (2.2.1) to be automatically a solution of (2.6) is that the strain-energy function of the material satisfies

$$
\phi_{2}(\omega)=D \phi_{1}(\omega)
$$

where $D$ is a constant which can depend upon $\lambda$. In the following sections we show for the general material which does not satisfy $(2.8)$ that the only possible consistent solutions of (2.2.1) and (2.6) are those of the form (2.7). 
It is convenient here to define the following functions,

$$
\begin{aligned}
& f(\omega)=\omega \phi_{1}(\omega), \\
& g(\omega)=\omega^{2} \phi_{2}(\omega),
\end{aligned}
$$

and we note that since $\phi_{1}(\omega)$ is essentially the generalized shear modulus of the material we shall assume throughout that $f(\omega)$ is never zero for non-constant $u(X, Y)$. We shall also frequently use the function $h(\omega)$ which we define by

$$
h(\omega)=\frac{g(\omega)}{\omega f(\omega)}=\frac{\phi_{2}(\omega)}{\phi_{1}(\omega)},
$$

and we mention that $h(\omega)$ constant gives rise to Knowles' criterion (2.8). In the following section we show that (2.6) can be expressed in a form which enables the sufficiency of $(2.8)$ to be established directly.

\section{Alternative derivation of the sufficiency of Knowles' criterion}

From (2.3) we can introduce the function $\theta(X, Y)$ such that

$$
u_{X}=\omega \cos \theta, \quad u_{Y}=\omega \sin \theta,
$$

and on equating expressions for $u_{X Y}$ we obtain

$$
\theta_{X} \cos \theta+\theta_{Y} \sin \theta=\frac{1}{\omega}\left[\omega_{Y} \cos \theta-\omega_{X} \sin \theta\right] .
$$

In addition from (2.2.1) and (3.1) we can deduce

$$
\theta_{X} \sin \theta-\theta_{X} \cos \theta=\frac{f^{\prime}(\omega)}{f(\omega)}\left[\omega_{X} \cos \theta+\omega_{Y} \sin \theta\right],
$$

where $f(\omega)$ is defined by (2.9.1) and we follow the convention throughout that primes denote differentiation with respect to the argument indicated. We now use (3.2) and (3.3) in the forms given to express (2.6) in an alternative form.

Using (3.1) we see that (2.6) becomes

$$
2 \frac{\partial^{2}}{\partial X \partial Y}[g(\omega) \cos 2 \theta]=\left(\frac{\partial^{2}}{\partial X^{2}}-\frac{\partial^{2}}{\partial Y^{2}}\right)[g(\omega) \sin 2 \theta],
$$

where $g(\omega)$ is defined by (2.9.2). If we now rewrite (3.4) as

$$
\begin{gathered}
\frac{\partial}{\partial X}\left\{\frac{\partial}{\partial Y}[g(\omega) \cos 2 \theta]-\frac{\partial}{\partial X}[g(\omega) \sin 2 \theta]\right\}+\frac{\partial}{\partial Y}\left\{\frac{\partial}{\partial X}[g(\omega) \cos 2 \theta]+\frac{\partial}{\partial Y}[g(\omega) \sin 2 \theta]\right\} \\
=0,
\end{gathered}
$$

and perform the differentiations inside the brackets in (3.5) then on eliminating the partial derivatives $\theta_{X}$ and $\theta_{Y}$ by means of (3.2) and (3.3) we can show in a 
straightforward manner that (3.5) becomes

$$
\begin{array}{r}
\frac{\partial}{\partial X}\left\{H(\omega)\left[\omega_{Y} \cos 2 \theta-\omega_{X} \sin 2 \theta\right]+g(\omega)\left[\frac{f^{\prime}(\omega)}{f(\omega)}-\frac{1}{\omega}\right] \omega_{Y}\right\} \\
+\frac{\partial}{\partial Y}\left\{H(\omega)\left[\omega_{X} \cos 2 \theta+\omega_{Y} \sin 2 \theta\right]-g(\omega)\left[\frac{f^{\prime}(\omega)}{f(\omega)}-\frac{1}{\omega}\right] \omega_{X}\right\}=0,
\end{array}
$$

where the function $H(\omega)$ is defined by

$$
H(\omega)=g^{\prime}(\omega)-g(\omega)\left[\frac{f^{\prime}(\omega)}{f(\omega)}+\frac{1}{\omega}\right] .
$$

If we again make use of (3.2) and (3.3) then from (3.6) we obtain

$$
\begin{aligned}
H(\omega) & {\left[2 \omega_{X Y} \cos 2 \theta+\left(\omega_{Y Y}-\omega_{X X}\right) \sin 2 \theta\right] } \\
+ & \left\{H^{\prime}(\omega)-H(\omega)\left[\frac{f^{\prime}(\omega)}{f(\omega)}+\frac{1}{\omega}\right]\right\}\left[2 \omega_{X} \omega_{Y} \cos 2 \theta+\left(\omega_{Y}^{2}-\omega_{X}^{2}\right) \sin 2 \theta\right]=0 .
\end{aligned}
$$

In terms of the function $h(\omega)$ defined by (2.10) we see on using (3.7) that (3.8) becomes

$$
\begin{aligned}
h^{\prime}(\omega)\left[2 \omega_{X Y} \cos 2 \theta\right. & \left.+\left(\omega_{Y Y}-\omega_{X X}\right) \sin 2 \theta\right] \\
& +h^{\prime \prime}(\omega)\left[2 \omega_{X} \omega_{Y} \cos 2 \theta+\left(\omega_{Y}^{2}-\omega_{X}^{2}\right) \sin 2 \theta\right]=0,
\end{aligned}
$$

and this is the alternative form of (2.6) from which the sufficiency of the criterion due to Knowles [5] can be deduced immediately. Evidently if the strain-energy function of the material is such that $h(\omega)$ is constant then (3.9) is satisfied trivially and in this case for every solution of (2.2.1) there always exists a function $q(X, Y)$ such that (2.2.2) and (2.2.3) are satisfied. For further details of the case of $h(\omega)$ constant the reader is referred to [5]. In the following section we suppose that $h(\omega)$ is not constant and we show not only the necessity of (2.8) but also that for the general material of this type the only consistent solutions of (2.2.1) and (2.6) are those of the form (2.7).

\section{The general material not satisfying Knowles' criterion}

In this section we suppose that $h^{\prime}(\omega)$ is non-zero and we remind the reader of our assumption noted at the end of Section 2 that $f(\omega)$ is never zero for nonconstant $u(X, Y)$. If we use (3.1) to eliminate $\theta(X, Y)$ from (3.9) then we obtain

$$
\begin{aligned}
& h^{\prime}(\omega)\left[\omega_{X Y}\left(u_{X}^{2}-u_{Y}^{2}\right)+\left(\omega_{Y Y}-\omega_{X X}\right) u_{X} u_{Y}\right] \\
& \quad+h^{\prime \prime}(\omega)\left[\omega_{X} \omega_{Y}\left(u_{X}^{2}-u_{Y}^{2}\right)+\left(\omega_{Y}^{2}-\omega_{X}^{2}\right) u_{X} u_{Y}\right]=0 .
\end{aligned}
$$


In terms of the Jacobian,

$$
\frac{\partial(u, \omega)}{\partial(X, Y)}=u_{X} \omega_{Y}-u_{Y} \omega_{X}
$$

we can show on using (2.3) that (4.1) becomes

$$
u_{X} \frac{\partial}{\partial X}\left[h^{\prime}(\omega) \frac{\partial(u, \omega)}{\partial(X, Y)}\right]+u_{Y} \frac{\partial}{\partial Y}\left[h^{\prime}(\omega) \frac{\partial(u, \omega)}{\partial(X, Y)}\right]=0 .
$$

From (2.2.1) we now introduce the function $v(X, Y)$ such that

$$
\phi_{1}(\omega) u_{X}=v_{Y}, \quad \phi_{1}(\omega) u_{Y}=-v_{X},
$$

and we note that from these relations and (2.3) and (2.9.1) we have

$$
\frac{\partial(u, v)}{\partial(X, Y)}=\omega f(\omega)
$$

From (4.3) and (4.4) we can deduce

$$
h^{\prime}(\omega) \frac{\partial(u, \omega)}{\partial(X, Y)}=F^{\prime}(v)
$$

where $F^{\prime}(v)$ is some arbitrary function of $v$ which for convenience we have expressed as a derivative with respect to $v$. If now we recall our assumption that $f(\omega)$ is never zero for non-constant $u(X, Y)$ then we see from (4.5) that we can treat $u$ and $v$ as independent variables so that on dividing (4.6) by (4.5) and using the usual rule for Jacobians we obtain

$$
\omega f(\omega) h^{\prime}(\omega) \frac{\partial \omega}{\partial v}=F^{\prime}(v)
$$

where we are evidently now considering $\omega$ as a function of $u$ and $v$. On integrating (4.7) we have

$$
\int_{\omega 0}^{\omega} t f(t) h^{\prime}(t) d t=F(v)+G(u),
$$

where $\omega_{0}$ is some arbitrary constant and $G(u)$ is an arbitrary function of $u$. We have thus established

$$
\omega=\Phi[F(v)+G(u)]
$$

where for a given material $\Phi$ is in principle a known function while both $F(v)$ and $G(u)$ are as yet undetermined arbitrary functions.

We now make use of the form (4.9) for $\omega$ to obtain the function $\theta(X, Y)$ defined by (3.1). For convenience we set

$$
\rho=F(v)+G(u)
$$


so that (4.7) becomes

$$
\omega f(\omega) h^{\prime}(\omega) \frac{d \omega}{d \rho}=1
$$

and it is important to emphasize again that for a given material $\omega$ as a function of $\rho$ is determined by (4.11). Using the form (4.9) for $\omega$ we can deduce from (3.2) and (3.3),

$$
\begin{aligned}
& \theta_{X}=\frac{f(\omega)}{\omega} \frac{d \omega}{d \rho} F^{\prime}(v) \cos \theta+\frac{\omega}{f(\omega)} \frac{d f(\omega)}{d \rho} G^{\prime}(u) \sin \theta, \\
& \theta_{Y}=\frac{f(\omega)}{\omega} \frac{d \omega}{d \rho} F^{\prime}(v) \sin \theta-\frac{\omega}{f(\omega)} \frac{d f(\omega)}{d \rho} G^{\prime}(u) \cos \theta,
\end{aligned}
$$

where we have made frequent use of (3.1) and (4.10) as well as the relations

$$
v_{X}=-f(\omega) \sin \theta, \quad v_{Y}=f(\omega) \cos \theta,
$$

which are obtained from (4.4), (3.1) and (2.9.1). On equating expressions for $\theta_{X Y}$ and making use of the above relations we obtain

$$
\frac{\partial}{\partial v}\left[\frac{f(\omega)}{\omega^{2}} \frac{d \omega}{d \rho} F^{\prime}(v)\right]+\frac{\partial}{\partial u}\left[\frac{\omega}{f(\omega)^{2}} \frac{d f(\omega)}{d \rho} G^{\prime}(u)\right]=0,
$$

which is the sole equation for the determination of the functions $F(v)$ and $G(u)$. Now since $u$ and $v$ are independent variables and for a given material $\omega$ and $f(\omega)$ are prescribed functions of $\rho$, the only possible way (4.14) can be satisfied without imposing a further restriction on the strain-energy function of the material is that if $\rho$ is either a function of $v$ only or a function of $u$ only. For special materials there are situations for which (4.14) can be satisfied with $\rho$ as a function of both $u$ and $v$ and these are discussed in the following section. For the general material not satisfying Knowles' criterion we now consider separately the solutions arising from the cases $\rho=F(v)$ and $\rho=G(u)$.

For the case of $\rho=F(v)$ we obtain from (4.14)

$$
\frac{f(\omega)}{\omega^{2}} \frac{d \omega}{d \rho} F^{\prime}(v)=\alpha
$$

where $\alpha$ is a constant and we note that in principle (4.15) together with (4.11) determines the function $F(v)$. From (3.1), (4.12) and (4.15) we find that $\theta(X, Y)$ is given by

$$
\theta=\alpha u+\beta
$$

where $\beta$ is a constant. 
Now using (4.15) and (4.16) we see that (3.1) and (4.13) become respectively

$$
\begin{aligned}
& u_{X}=\omega \cos (\alpha u+\beta), \quad u_{X}=\omega \sin (\alpha u+\beta), \\
& \omega_{X}=-\alpha \omega^{2} \sin (\alpha u+\beta), \quad \omega_{Y}=\alpha \omega^{2} \cos (\alpha u+\beta),
\end{aligned}
$$

and we note that we have

$$
\frac{\partial(u, \omega)}{\partial(X, Y)}=\alpha \omega^{3}
$$

Assuming $\alpha$ is non-zero and considering $X$ and $Y$ as functions of $u$ and $\omega$ we have in the usual way from (4.17) and (4.18)

$$
\begin{aligned}
& \frac{\partial Y}{\partial \omega}=\frac{1}{\alpha \omega^{2}} \cos (\alpha u+\beta), \quad \frac{\partial X}{\partial \omega}=-\frac{1}{\alpha \omega^{2}} \sin (\alpha u+\beta), \\
& \frac{\partial Y}{\partial u}=\frac{1}{\omega} \sin (\alpha u+\beta), \quad \frac{\partial X}{\partial u}=\frac{1}{\omega} \cos (\alpha u+\beta),
\end{aligned}
$$

and these equations can be integrated to yield

$$
X=\frac{1}{\alpha \omega} \sin (\alpha u+\beta)+X_{0}, \quad Y=-\frac{1}{\alpha \omega} \cos (\alpha u+\beta)+Y_{0},
$$

where $X_{0}$ and $Y_{0}$ are arbitrary constants. On inverting (4.20) we obtain

$$
\begin{aligned}
& u(X, Y)=\frac{1}{\alpha} \tan ^{-1}\left(\frac{Y-Y_{0}}{X-X_{0}}\right)-\frac{1}{\alpha}\left(\beta+\frac{\pi}{2}\right), \\
& \omega(X, Y)=\frac{1}{\alpha}\left[\left(X-X_{0}\right)^{2}+\left(Y-Y_{0}\right)^{2}\right]^{-\frac{1}{t}},
\end{aligned}
$$

and the reader can verify that (4.21.1) and (4.21.2) are consistent with (2.3). Apart from the arbitrary constants (4.21) is evidently the deformation (i) of (2.7). The reader can easily verify from (4.17) that the case of $\alpha$ zero gives rise to the deformation (ii) of (2.7).

The case of $\rho=G(u)$ is similar to the above. From (4.14) we have

$$
\frac{\omega}{f(\omega)^{2}} \frac{d f(\omega)}{d \rho} G^{\prime}(u)=-\gamma
$$

where $\gamma$ is a constant and we find from this equation and (4.12) and (4.13) that $\theta(X, Y)$ is given by

$$
\theta=\gamma v+\delta
$$

where $\delta$ is a further arbitrary constant. 
On using (4.22) and (4.23) we have from (4.13) and (3.1)

$$
\begin{aligned}
& v_{X}=-f \sin (\gamma v+\delta), \quad v_{Y}=f \cos (\gamma v+\delta), \\
& f_{X}=-\gamma f^{2} \cos (\gamma v+\delta), \quad f_{Y}=-\gamma f^{2} \sin (\gamma v+\delta) .
\end{aligned}
$$

If in this case we consider $X$ and $Y$ as functions of $v$ and $f$ then for $\gamma$ non-zero we can show as for the previous case that $X(v, f)$ and $Y(v, f)$ are given by

$$
X=\frac{1}{\gamma f} \cos (\gamma v+\delta)+X_{0}, \quad Y=\frac{1}{\gamma f} \sin (\gamma v+\beta)+Y_{0},
$$

where again $X_{0}$ and $Y_{0}$ are arbitrary constants. Thus in particular from (4.25) we have

$$
f(\omega)=\frac{1}{\gamma}\left[\left(X-X_{0}\right)^{2}+\left(Y-Y_{0}\right)^{2}\right]^{-\frac{1}{2}},
$$

and since $\omega$ is a function of $u$ we have the deformation (iii) of (2.7). If $\gamma$ is zero then from (4.24) we see that the deformation is such that $f$ is constant and from (3.1) and (4.23) we have

$$
u_{X}=\omega \cos \delta, \quad u_{Y}=\omega \sin \delta .
$$

On solving the partial differential equation for $\omega$ which results from (4.27) on equating expressions for $u_{X Y}$ we obtain a solution of the form

$$
\omega(X, Y)=\Psi(X \cos \delta+Y \sin \delta),
$$

where $\Psi$ is an arbitrary function. From (4.27) and (4.28) we see that $u(X, Y)$ is of the same form as the deformation (iv) of (2.7).

We have thus established the necessity of (2.8) and in addition for the general material which does not satisfy Knowles' criterion we have shown that the only possible consistent solutions of (2.2.1) and (2.6) are those of the form (2.7). In the following section we briefly discuss the special materials which admit solutions $\rho$ of (4.14) which are functions of both $u$ and $v$.

\section{Special materials}

If we are at liberty to choose the functions $f(\omega)$ and $h(\omega)$ then it is possible to find solutions $\rho$ of (4.14) which are functions of both $u$ and $v$. The resulting special materials although perhaps physically well defined are somewhat contrived and appear not to include any standard materials which have been proposed in the literature. We will therefore not discuss all of these situations in any great detail. The following list of expressions for $\rho$, which may not be exhaustive, are possible 
solutions of (4.14) for particular strain-energy functions:

(a) $\rho=A u+B v$,

(b) $\rho=A \log (v / u)+B$,

(c) $\rho=A u^{2}+B v^{2}$,

where $A$ and $B$ denote arbitrary constants which can if necessary be functions of the constant $\lambda$.

The first case is interesting since all the mathematical details can be effected in relatively simple terms and an explicit form for the function $u(X, Y)$ can be deduced. Moreover, in this case we find that the constraint on the response coefficients can be considered as a generalization of Knowles' criterion (2.8). For the case (a) we find that (4.14) admits a first integral which on using (4.11) can be integrated again to yield

$$
h(\omega)=D+C\left[\frac{A^{2}}{f(\omega)^{2}}+\frac{B^{2}}{\omega^{2}}\right],
$$

where $C$ and $D$ are integration constants which can depend upon $\lambda$. In terms of the response coefficients $\phi_{1}(\omega)$ and $\phi_{2}(\omega)$ we see from (2.9.1) and (2.10) that (5.2) becomes

$$
\phi_{2}(\omega)=D \phi_{1}(\omega)+\frac{C}{\omega^{2}}\left[\frac{A^{2}}{\phi_{1}(\omega)}+B^{2} \phi_{1}(\omega)\right],
$$

which evidently can be considered to be a generalization of Knowles' criterion (2.8). From (3.1), (4.12), (4.13) and (5.1) (a) we obtain the relations,

$$
\begin{aligned}
& \theta_{X}=\frac{d \omega}{d \rho}\left[\frac{f(\omega)}{\omega} B \cos \theta+\omega \frac{f^{\prime}(\omega)}{f(\omega)} A \sin \theta\right], \\
& \theta_{Y}=\frac{d \omega}{d \rho}\left[\frac{f(\omega)}{\omega} B \sin \theta-\omega \frac{f^{\prime}(\omega)}{f(\omega)} A \cos \theta\right], \\
& \omega_{X}=\frac{d \omega}{d \rho}[\omega A \cos \theta-f(\omega) B \sin \theta], \\
& \omega_{Y}=\frac{d \omega}{d \rho}[\omega A \sin \theta+f(\omega) B \cos \theta] .
\end{aligned}
$$

Now from (5.4) and the first integral obtained from (4.14) we have

$$
\frac{\partial(\theta, \omega)}{\partial(X, Y)}=-\frac{\omega f(\omega)}{2 C} \frac{d \omega}{d \rho},
$$


and if we consider $X$ and $Y$ as functions of $\theta$ and $\omega$ then as described in the previous section we can deduce from (5.4) and (5.5) that $X(\theta, \omega)$ and $Y(\theta, \omega)$ are given by

$$
\begin{aligned}
& X=2 C\left[\frac{A}{f(\omega)} \cos \theta-\frac{B}{\omega} \sin \theta\right]+X_{0}, \\
& Y=2 C\left[\frac{A}{f(\omega)} \sin \theta+\frac{B}{\omega} \cos \theta\right]+Y_{0},
\end{aligned}
$$

where $X_{0}$ and $Y_{0}$ are arbitrary constants. From (5.2) and (5.6) we have

$$
h[\omega(R)]=D+\frac{R^{2}}{4 C}
$$

where $R$ is defined by

$$
R=\left[\left(X-X_{0}\right)^{2}+\left(Y-Y_{0}\right)^{2}\right]^{\frac{1}{2}},
$$

and for a given material satisfying (5.3), equation (5.7) defines $\omega$ as a function of $R$. If we use (5.6) to obtain expressions for $\sin \theta$ and $\cos \theta$ then from (3.1) we can deduce that $u(X, Y)$ is given by

$$
u(X, Y)=-2 B C \tan ^{-1}\left[\frac{Y-Y_{0}}{X-X_{0}}\right]+2 A C \int_{R_{0}}^{R} \frac{d t}{t \phi_{1}[\omega(t)]}+u_{0}
$$

where $R_{0}$ and $u_{0}$ are arbitrary constants. We note that, as would be expected, the cases $A=0$ and $B=0$ yield respectively deformations (i) and (iii) of (2.7). We remark that case (b) of (5.1) is similar to (a) in that (4.14) admits a first integral. However, the details are not simple and we shall not discuss this case further.

For case (c) we consider the material with functions $f(\omega)$ and $h(\omega)$ defined by

$$
f(\omega)=\frac{\alpha \omega}{\left(\beta \omega^{2}+\gamma\right)^{\frac{1}{2}}}, \quad h(\omega)=\frac{\delta}{\omega^{2}}+\varepsilon,
$$

where $\alpha, \beta, \gamma, \delta$ and $\varepsilon$ are constants which can be functions of $\lambda$. Equation (5.10) can be shown to give rise to a strain-energy function of the form,

$$
\Sigma(\lambda, \omega)=2 \frac{\alpha}{\beta}\left(\beta \omega^{2}+\gamma\right)^{\natural}+\Sigma_{0}
$$

provided that $\delta$ and $\varepsilon$ are given respectively by

$$
\begin{aligned}
& \delta=2\left(\lambda^{3}-1\right)-2 \lambda^{2} \frac{\gamma}{\alpha}\left(\frac{\alpha}{\beta}\right)^{\prime}-\lambda^{2} \frac{\gamma^{\prime}}{\beta}, \\
& \varepsilon=\lambda^{2}\left(\frac{\beta^{\prime}}{\beta}-2 \frac{\alpha^{\prime}}{\alpha}\right),
\end{aligned}
$$

where primes here denote differentiation with respect to $\lambda$ and $\Sigma_{0}$ is a constant 
which is independent of $\lambda$. From (4.11) and (5.10) we can deduce the relations

$$
\begin{aligned}
\frac{1}{\omega} & =\left(\frac{\beta}{\gamma}\right)^{\frac{t}{t}} \sinh \left(\frac{\gamma^{\frac{\hbar}{}} \rho}{2 \alpha \delta}+\rho_{0}\right), \\
\frac{1}{f(\omega)} & =\frac{\beta^{\frac{1}{2}}}{\alpha} \cosh \left(\frac{\gamma^{\frac{1}{2} \rho}}{2 \alpha \delta}+\rho_{0}\right),
\end{aligned}
$$

where $\rho_{0}$ is an integration constant. For this special material we find for case (c) that (4.14) is satisfied provided the constants $A$ and $B$ are such that

$$
B=-\frac{\gamma}{\alpha^{2}} A,
$$

and from (3.1), (4.12) and (4.13) we can deduce that $\theta(X, Y)$ is given by

$$
\theta=\frac{\gamma A}{\alpha^{2} \delta} u v+\theta_{0}
$$

where $\theta_{0}$ is a constant. If we make use of the above equations then from (3.1), (4.5) and (4.13) we can show after a long but straightforward calculation that $X$ and $Y$ as functions of $u$ and $v$ are given by

$$
\begin{aligned}
& X(u, v)=\left(\frac{\beta}{\gamma}\right)^{\frac{1}{2}} \operatorname{Re}\left\{\int_{s_{0}}^{s} \sinh \left(C t^{2}+\sigma\right) d t\right\}+X_{0}, \\
& Y(u, v)=\left(\frac{\beta}{\gamma}\right)^{\frac{1}{t}} \operatorname{Im}\left\{\int_{s_{0}}^{s} \cosh \left(C t^{2}+\sigma\right) d t\right\}+Y_{0},
\end{aligned}
$$

where $s_{0}, X_{0}$ and $Y_{0}$ are further arbitrary constants. The constants $C$ and $\sigma$ are defined by

$$
C=\frac{\gamma^{\frac{1}{1}} A}{2 \alpha \delta}, \quad \sigma=\rho_{0}+i \theta_{0},
$$

and the complex variable $s$ is given by

$$
s=u+i \frac{\gamma^{\frac{3}{2}}}{\alpha} v
$$

Evidently in this case $u$ is not a simple function of $X$ and $Y$. It is however well defined as an implicit function by the relations (5.16). We note that for suitably chosen constants the functions $f(\omega)$ and $h(\omega)$ defined by (5.10) satisfy the constraint (5.2) obtained for case (a).

Finally, in this section we remark that if $f(\omega)$ is identically constant for all deformations of the form (2.1) then (4.14) admits solutions $\rho$ which are functions of both $u$ and $v$. However, for $f(\omega)$ constant we can show that the strain-energy function $\Sigma$ must be a linear function of $\omega$ and thus this material is not physically meaningful since $\Sigma$ must be an even function of $\omega$. 


\section{Conclusion}

For generalized shear deformations (2.1) of isotropic incompressible hyperelastic materials we have shown for the general material not satisfying Knowles' criterion (2.8) that the only consistent solutions $u(X, Y)$ of (2.2.1) and (2.6) are those of the form (2.7) which are known to be well defined for all strain-energy functions. We have eliminated the possibility of obtaining further solutions of this type since there can be no solutions $\rho$ of (4.14) which are functions of both $u$ and $v$ without imposing further restrictions on the strain-energy function of the material. In deriving these results we have also obtained an alternative derivation of Knowles' criterion, which if satisfied ensures that every solution of (2.2.1) is automatically a solution of (2.6). Moreover, we have also briefly indicated how solutions $\rho$ of (4.14) which are functions of both $u$ and $v$ can be obtained for very special materials. The most illuminating case of this being (5.1) (a) for which $u(X, Y)$ is given explicitly by (5.9). In this case the restriction (5.3) on the response coefficients can be considered to generalize Knowles' criterion (2.8).

In summary for those materials not satisfying Knowles' criterion such as those proposed in [1], [2] and [3] the only generalized shear deformations are those of the form (2.7) and more general types of these deformations are only applicable to very special materials which do not include any of the standard experimentally determined prototypes for rubber-like materials.

\section{Acknowledgements}

The writer is indebted to Dr. V. G. Hart for encouraging this work and also to Professor J. K. Knowles and Dr. R. V. Nillsen for their helpful comments.

\section{REFERENCES}

[1] H. Alexander, "A constitutive relation for rubber-like materials", Int. J. Engng. Sci. 6 (1968), 549-563.

[2] A. N. Gent and A. G. Thomas, "Forms for the stored (strain) energy functions for vulcanized rubber", J. of Polym. Sci. 28 (1958), 625-628.

[3] L. J. Hart-Smith, "Elasticity parameters for finite deformations of rubber-like materials", Z. angew. Math. Phys. 17 (1960), 608-626.

[4] J. M. Hill, "Partial solutions of finite elasticity-Three dimensional deformations", Z. angew. Math. Phys. 24 (1973), 609-618.

[5] J. K. Knowles, "Anti-plane strain deformations for isotropic incompressible hyperelastic materials", J. Austral. Math. Soc. 19 (Series B), (1977), 400-415.

Department of Mathematics

University of Wollongong

Australia 